Trichomonas vaginalis (TV) by APTIMA TV on a TIGRIS instrument. Each patient was asked on a scale of one to five to express strength of agreement or disagreement with ease or comfort of the 6 step self collection process for the VSCT.

Results There were a total of 22 CT, 19 TV and 2 NG infections with dual infections in 6 people (one CT and NG, one NG and TV and four CT and TV). Prevalences were as follows: CT 3.9\% (GYC 1.3\% and YHC $12.6 \%$ ); NG $0.3 \%$ (2.0\% in YHC); TV $3.4 \%$ (GYC $0.4 \%$ and YHC 13.4\%). Sensitivity for CT infections were CSCT 100\%, PC 100\%, SP 81.8\%; VSCT-self 100\%, VSCT-physician 95.4\%: for TV infections CSCT $89.4 \%$, PC $84.2 \%$, SP $63.2 \%$; both VSCT $100 \%$ : for NG all collections $100 \%$. There were no false positives (\% specificity 100). Results of the survey revealed that the majority of patients found opening the package, self sampling, insertion of the SCT swab into preservation media and uncapping and recapping the tube were relatively easy to perform. Eighty two per cent experienced no discomfort using the SCT kit for collection.

Conclusions APTIMA testing on the TIGRIS showed that the cervical and vaginal SCT and PC L-Pap samples were 100\% sensitive in detecting CT and NG infections. VSCT samples detected all TV infections but cervical SCT and PC L-Pap were less sensitive. SP LPap samples showed reduced sensitivity for CT and TV. Overall, patients were in strong agreement with the ease of use of the VSCT and most found the process comfortable.

\section{3-S3.02 PERFORMANCE OF THE BIO-RAD DX CT/NG/MG ASSAY FOR SIMULTANEOUS DETECTION OF CHLAMYDIA TRACHOMATIS, NEISSERIA GONORRHOEAE AND MYCOPLASMA GENITALIUM IN UROGENITAL SAMPLES}

doi:10.1136/sextrans-2011-050109.116

${ }^{1} \mathrm{C}$ Bébéar, ${ }^{1}$ Chloe Le Roy, ${ }^{2}$ ssabelle Le Hen, ${ }^{1} \mathrm{M}$ Clerc, ${ }^{2} \mathrm{~V}$ Arfel, ${ }^{2} \mathrm{~F}$ Normandin, ${ }^{1} \mathrm{~B}$ de Barbeyrac. 'INRA-Université de Bordeaux2, Bordeaux, France; ${ }^{2}$ Maison départementale de la santé, Bordeaux, France

Objectives To investigate the performance of the Bio-Rad Dx CT/ NG/MG Assay with an internal control for the detection of Chlamydia trachomatis (CT) and Mycoplasma genitalium (MG) in urogenital samples in comparison with the Roche Cobas TaqMan CT test and an in-house TaqMan PCR test for MG. For Neisseria gonorrhoeae (NG), only positive PCR results were controlled by culture.

Methods In this prospective study, urogenital samples were obtained from symptomatic and asymptomatic patients attending the STI center of Bordeaux, France, from January to April 2010. For symptomatic women and men, two endocervical swabs and two urethral swabs were collected, respectively. All patients and women collected first-catch urines and two vaginal swabs, respectively. Two swabs per site were used, a flocked swab in the universal transport medium and the Bio-Rad flocked swab in its transport medium. For the Bio-Rad CT/NG/MG assay, the DNA was manually extracted and amplified according to the manufacturer's instructions. For the comparator PCR tests, DNA was extracted using the MagNa Pure LC instrument (Roche Diagnostics) and amplified with the Cobas TaqMan CT 48 assay (Roche Diagnostics) and with a MgPa-targeted PCR assay on an ABI Prism 7000 (Applied Biosystems) for MG. The patient was considered as infected if at least two of the 4 or 6 PCR tests performed according to the gender and characteristics of patients, were positive. For asymptomatic men, in case of discrepancy, the urine sample was retested by both methods and the patient was considered infected if at least two of the four PCR results were positive for the considered microorganism.

Results A total of 658 clinical specimens (259 male and 180 female urines, 191 vaginal, 21 cervix and seven urethral swabs) from 453 patients were analysed. The prevalence of CT and MG infections was $7.7 \%(20 / 260)$ and $1.9 \%(5 / 260)$ in men and $10.3 \%(20 / 193)$ and
2\% (4/193) in women, respectively. The Bio-Rad Dx CT/NG/MG test sensitivity was $100 \%$ for CT and MG in men and women. In male urines, the specificity was $99.6 \%$ for CT and $100 \%$ for MG. In women, the specificity was $99.5 \%$ for swabs and $100 \%$ for urines for CT and MG. All 7 NG-PCR positive samples were positive by culture. Patients were co-infected in 5/56 (9\%) with CT/MG in three cases and CT/NG in two cases.

Conclusion The Bio-Rad Dx CT/NG/MG Assay was found to be very effective for the simultaneous detection of CT, MG, and NG infections in urogenital specimens.

\section{3-S3.03 DIFFERING NEISSERIA GONORRHOEAE BACTERIAL LOADS IN THE PHARYNX AND RECTUM: IMPLICATIONS FOR GONOCOCCAL DETECTION, TRANSMISSION AND CONTROL}

doi:10.1136/sextrans-2011-050109.117

${ }^{1} \mathrm{M}$ Bissessor, ${ }^{2} \mathrm{~S}$ Tabrizi, ${ }^{1} \mathrm{C}$ Fairley, ${ }^{2} \mathrm{~J}$ Danielewski, ${ }^{1} \mathrm{~B}$ Whitton, ${ }^{1} \mathrm{~S}$ Bird, ${ }^{2} \mathrm{~S}$ Garland, ${ }^{1} \mathrm{M}$ Chen. ${ }^{1}$ Melbourne Sexual Health Centre Carlton, Australia; ${ }^{2}$ Royal Womens hospital, Australia

Background To help improve our understanding of the potential transmissibility of gonococcal infections from the pharynx and rectum we measured gonococcal bacterial loads at these sites and examined clinical and laboratory determinants of these loads.

Methods Men who had sex with men were tested for pharyngeal and rectal gonorrhoea by culture using modified Thayer Martin medium and by two real-time quantitative qPCRs targetting opa gene, and porA pseudogene.

Results 1011 rectal and 1076 pharyngeal specimens were obtained from 1076 MSM. Forty three (3.9\%) pharyngeal specimens were PCR positive of which 17 were culture positive (sensitivity $39 \%$, $95 \%$ CI: $25 \%$ to $54 \%$ ). Forty seven (4.6\%) rectal specimens were PCR positive of which 25 were also culture positive (sensitivity $58 \%, 95 \%$ CI $44 \%$ to $71 \%$ ). The median bacterial load among PCR positive rectal infections (18960 copies per swab) was significantly higher than that for PCR positive pharyngeal infections (2,100 copies per swab) $(p=0.001)$. The median bacterial load among men with symptomatic rectal infection was higher (278 800 copies per swab) than with asymptomatic men, PCR positive rectal infections $(13,980$ copies per swab, $p<0.001)$. The median bacterial load of gonorrhoea was significantly higher in culture positive than culture negative specimens. This applied for both rectal $(p<0.001)$ as well as pharyngeal infections $(p=0.03)$.

Conclusion Higher bacterial loads of gonorrhoea were observed in rectal infections, particularly with symptomatic rectal infections. This has implications for gonococcal transmission and control.

\section{3-S3.04 SELF-ADMINISTERED NEISSERIA GONORRHEAE AND CHLAMYDIA TRACHOMATIS TESTING IN THE PHARYNX AND RECTUM AMONG MEN WHO HAVE SEX WITH MEN IN WASHINGTON, DC}

doi:10.1136/sextrans-2011-050109.118

${ }^{1} \mathrm{M}$ Sexton, ${ }^{2} \mathrm{~J}$ Baker, ${ }^{3} \mathrm{R}$ Perkins, ${ }^{4} \mathrm{~K}$ Nakagawa, ${ }^{3} \mathrm{~B}$ Jucha, ${ }^{3} \mathrm{D}$ Baker, ${ }^{5} \mathrm{R}$ Slack, ${ }^{6} \mathrm{~S}$ Arora, ${ }^{5} \mathrm{M}$ Plankey. ${ }^{1}$ Emory University School of Medicine, Atlanta, USA; ${ }^{2}$ Fenway Institute, Boston, USA; ' Whitman Walker Clinic, Washington, USA; ${ }^{3}$ UC Davis School of Medicine, Sacramento, USA; ${ }^{5}$ Georgetown University Medical Center, Washington, USA; ${ }^{6}$ Johns Hopkins University, School of Public Health, Baltimore, USA

Background Recent studies have demonstrated a high prevalence of pharyngeal (P) and rectal (R) Neisseria gonorrheae (GC) and Chlamydia trachomatis (CT) infections among men who have sex with men (MSM), which is concerning given the potential for harmful 